\title{
Krylov-projected quantum Monte Carlo
}

\author{
N. S. Blunt, ${ }^{*}$ Ali Alavi, and George H. Booth ${ }^{\dagger}$ \\ University Chemical Laboratory, Lensfield Road, Cambridge, CB2 1EW, United Kingdom \\ Max Planck Institute for Solid State Research, Heisenbergstraße 1, 70569 Stuttgart, Germany and \\ Department of Physics, King's College London, The Strand, London, WC2R 2LS, U.K.
}

(Dated: August 4, 2015)

\begin{abstract}
We present an approach to the calculation of arbitrary spectral, thermal and excited state properties within the full configuration interaction quantum Monte Carlo framework. This is achieved via an unbiased projection of the Hamiltonian eigenvalue problem into a space of stochastically sampled Krylov vectors, thus enabling the calculation of real-frequency spectral and thermal properties and avoiding explicit analytic continuation. We use this approach to calculate temperature-dependent properties and one- and two-body spectral functions for various Hubbard models, as well as isolated excited states in ab initio systems.
\end{abstract}

Quantum Monte Carlo (QMC) in its various guises, is undoubtedly one of the most important approaches for accurate elucidation of properties for correlated systems [1-5]. However, these successes have focused primarily on the ground state energy and observables which commute with the Hamiltonian. Critical importance for a deeper understanding of correlated systems comes from dynamic correlation functions and spectral quantities. These mirror how we perceive our environment, namely by perturbing a system and measuring its response - the basis of nearly all spectroscopic and experimental approaches. This gives us direct insight into optical, magnetic and other beyond-ground-state properties, and allow for direct comparison to experimental results.

Direct access to dynamic properties is a persistent difficulty for QMC approaches in general. While in the absence of a sign problem, unbiased imaginary-time spectra can be obtained[6-8], the analytic continuation to physical, real-frequency functions is notoriously ill-conditioned and can lead to artefacts and smoothing of features.[9] For more general Fermionic systems, higher temperatures must be simulated to alleviate the sign problem[10], while nodal constraints bias towards a particular solution and are difficult to extend to spectra $[1,7,11]$. Alternatively, projections into effective Hamiltonians have been able to obtain a few low-energy states, but again these are isolated states rather than practical approaches for thermal or spectral quantities[12, 13], while a modification of the propagator can lead to debilitating timestep issues[14].

Here, we present a new QMC approach for computing dynamic correlation functions, temperature-dependent quantities and isolated excited states for correlated quantum systems, even in the presence of a sign-problem. These correlation functions are unbiased in the limit of large averaging, and exact in the limit of large walker number. This is achieved by extending the recently developed Full Configuration Interaction Quantum Monte Carlo (FCIQMC) method[2, 15, 16], by combining it with ideas from the dynamical and finite-temperature Lanczos (FTLM) methods.[17-19] The key advantage of the approach is that it avoids any explicit storage over the full Hilbert space, instead only storing occupied states in the

\footnotetext{
* nsb37@cam.ac.uk

$\dagger$ george.booth@kcl.ac.uk
}

discretized wavefunction at each snapshot. This allows for sparsity in the wavefunction to be exploited to minimize memory bottlenecks, which are a primary limitation in conventional approaches which require explicit storage over the space[17, 18, 20, 21]. The result is a QMC method which although weakly exponentially scaling, in common with the ground state FCIQMC approach, can allow for systems to be treated well outside that possible by conventional means, and retains many of the important features of the parent method[15, 22]. These include a cancellation algorithm to ameliorate the sign problem, an absence of time-step error and large-scale parallelism.

An arbitrary dynamic correlation function is defined as

$$
G(\omega)=\left\langle\Psi_{0}\left|\hat{A}^{\dagger} \frac{1}{\omega-\left(\hat{H}-E_{0}\right)+i \eta} \hat{V}\right| \Psi_{0}\right\rangle,
$$

where $\hat{H}$ represents the Hamiltonian of the system, $\left\{\left|\Psi_{0}\right\rangle ; E_{0}\right\}$ is the ground state wavefunction and energy, $\eta$ is a small broadening parameter, and $\hat{V}$ and $\hat{A}$ are arbitrary operators which define the perturbation and observed quantity in the correlation function. In the case of these operators being single annihilation and creation operators one obtains the single-particle Green function, defining the system bandstructure and density of states.

The aim of our method is to stochastically obtain a projection of the Hamiltonian from the complete Hilbert space to an effective, reduced dimensionality space, such that it spans the degrees of freedom required to accurately describe the desired spectral or thermal quantity. In this work, we use a set of stochastically sampled wavefunctions from a FCIQMC calculation to define this transformation. If the initial state of the calculation is a stochastic representation of the wavefunction $\hat{V}\left|\Psi_{0}\right\rangle$, then propagation from this state[23] to the ground state will in principle span all states required to represent the expression in Eq. 1, equivalent to the space of ground state and all imaginary-time response vectors. Once the Hamiltonian is projected into this space, it can be exactly diagonalized, and the desired correlation function of Eq. 1 directly constructed in this eigenbasis - the Lehmann representation. For thermal quantities the approach is analogous, with the initial vector taken from the infinite-temperature distribution. Similar themes have been explored within continuum QMC, but applied to accelerate convergence for ground state properties[24]. 
Method:- An FCIQMC iteration consists of stochastically applying a projection operator, $\boldsymbol{P}$, to a walker distribution, denoted at iteration $i$ by $\boldsymbol{q}_{i}$, such that exact projection is achieved on average, whose distributions we denote as $\boldsymbol{\psi}_{i}$ [23]. The aim is to stochastically sample the Krylov subspace $\left\{\boldsymbol{\psi}_{0}, \boldsymbol{P} \boldsymbol{\psi}_{0}, \ldots, \boldsymbol{P}^{n-1} \boldsymbol{\psi}_{0}\right\}$. In projector QMC approaches one samples from the large $n$ limit of this subspace, which converges to the ground state. However, to obtain finite-temperature and dynamic quantities, the aim is now to stochastically project the Hamiltonian into the whole sampled Krylov subspace, which represents an efficient span of all states of interest, provided that $\boldsymbol{q}_{0}$ is chosen appropriately.

By averaging the FCIQMC walker amplitudes, the results of an exact propagation are rigorously approached for expectation values which depend linearly on the wavefunction $[15,16]$. In this work, quadratic quantities are required, but now $E\left[\boldsymbol{q}_{i}^{\dagger} \boldsymbol{q}_{j}\right] \neq \boldsymbol{\psi}_{i}^{\dagger} \boldsymbol{\psi}_{j}$, due to correlations between walker amplitudes, where $E\left[\boldsymbol{q}_{i}\right]$ denotes the expectation value. To compute these, two independent replica sets of walkers are propagated simultaneously (indexed via superscripts), such that the amplitudes are uncorrelated between them $[25,26]$, allowing for unbiased estimates of $\boldsymbol{\psi}_{i}^{\dagger} \boldsymbol{\psi}_{j}$ as $E\left[\boldsymbol{q}_{i}^{1 \dagger} \boldsymbol{q}_{j}^{2}\right]$ or $E\left[\boldsymbol{q}_{i}^{2 \dagger} \boldsymbol{q}_{j}^{1}\right]$. This approach for static correlation functions has been found to scale without difficulty within FCIQMC[27].

At selected iterations in an FCIQMC calculation the walker distribution is stored[28], and the overlap $(\boldsymbol{S})$ and Hamiltonian $(\boldsymbol{T})$ matrices between these subspace vectors calculated as

$$
\begin{aligned}
& S_{i j}=\left(\boldsymbol{q}_{i}^{1 \dagger} \boldsymbol{q}_{j}^{2}+\boldsymbol{q}_{i}^{2 \dagger} \boldsymbol{q}_{j}^{1}\right) / 2, \\
& T_{i j}=\left(\boldsymbol{q}_{i}^{1 \dagger} \boldsymbol{H} \boldsymbol{q}_{j}^{2}+\boldsymbol{q}_{i}^{2 \dagger} \boldsymbol{H} \boldsymbol{q}_{j}^{1}\right) / 2 .
\end{aligned}
$$

Whilst the overlap matrix estimate is trivial, calculating the $\boldsymbol{T}$ matrix exactly is expensive, and so instead it is stochastically sampled in the same manner as spawning steps in FCIQMC[27]. Thus, a simulation provides an estimate of the overlap matrix and the projected Hamiltonian in the basis of Krylov vectors chosen, and so we denote the method Krylov Projected (KP)-FCIQMC. Averaging these quantities over independent simulations can reduce errors in an unbiased manner, resulting in a generalised eigenvalue problem for the projected Hamiltonian. This can be solved by standard techniques (see supplementary material[29-31]). Many of the eigenvalues of $\boldsymbol{S}$ will be very small (or even negative within stochastic errors), since the sampled space becomes increasingly linearly dependent with continued propagation. We therefore discard these vectors of $\boldsymbol{S}$ without substantial loss of information. We refer to the eigenvectors which are kept as Löwdin vectors. We note that although the estimates of $\boldsymbol{T}$ and $\boldsymbol{S}$ are unbiased, the final eigenvalues will not be because eigenvalues are non-linear functions of these matrices. However, this bias can be systematically reduced with further averaging of $\boldsymbol{T}$ and $\boldsymbol{S}$.

For exact propagation with $\hat{P}=\hat{H}$, our approach will yield results identical to the Lanczos method. However, because the method exploits sparsity via a stochastic representation of the wavefunctions, large calculations can often use significantly less memory than an equivalent
Lanczos calculation, as has been the case for ground-state FCIQMC. Although our approach is in theory systematically improvable to exactness for the entire frequency range, in practice this becomes increasingly difficult for higher energy excitations. This is because high-energy excitations have a small component in the Krylov vectors, which decreases exponentially with imaginary time. This renders them particularly difficult to sample and susceptible to stochastic error in the sampled matrices. Despite this limitation, the approach can nevertheless be expected to obtain near-exact spectra for low-energy excitations in systems out of reach of traditional dynamical Lanczos approaches.

Finite-temperature:- We assess the method with the half-filled Hubbard model (defined in supplementary information) [16, 22]. Within the FTLM, thermal expectation values are computed via

$$
\operatorname{Tr}\left(e^{-\beta \hat{H}} \hat{A}\right)=\sum_{n=1}^{N} \sum_{i=0}^{M-1} e^{-\beta E_{i}^{n}}\left\langle n \mid \psi_{i}^{n}\right\rangle\left\langle\psi_{i}^{n}|\hat{A}| n\right\rangle+\mathcal{O}\left(\beta^{M}\right),
$$

where $|n\rangle$ labels a state in the $N$-dimensional Hilbert space, and $i$ labels the $M$ states of an eigensystem $\left\{\left|\psi_{i}^{n}\right\rangle ; E_{i}^{n}\right\}$ resulting from a Lanczos subspace with initial state $|n\rangle$. Thus, by performing $N$ Lanczos calculations consisting of $M-1$ applications of $\hat{H}$ each, one can obtain thermal quantities which are correct to order $\beta^{M-1}$. $N$ can be very large for systems of interest and so in practice one starts from a much smaller number of states, $R \ll N$, chosen as a random linear combination of all basis states, $|r\rangle=\sum_{n} \eta_{r n}|n\rangle$. This turns out to converge quickly with $R$, particularly at high temperatures[17, 18, 32-34]. In our stochastic approach the initial random vectors are created by distributing a given number of walkers randomly throughout the Hilbert space with coefficients \pm 1 . These initial states represent stochastic snapshots of the high-temperature limit which is exactly reproduced in the limit of large $R$.

As an initial test, Figure 1 presents the temperaturedependent energy, $E(\beta)$, in the one-dimensional 12-site Hubbard model at $U / t=1$. Including all symmetry sectors the Hilbert space dimension is $\approx 3 \times 10^{6}$, with the largest symmetry sector containing $\approx 7 \times 10^{4}$ determinants. However, the system is significantly undersampled with only $2 \times 10^{3}$ walkers used throughout, with the projected Hamiltonian and overlap matrices averaged over 10 calculations for each initial vector, $|r\rangle$. All symmetry sectors were obtained in one calculation, rather than symmetry-blocking Eq. 4, resulting in a choice of $R=1250$, while the number of Krylov vectors used was $M=20$, with 8 Löwdin vectors kept to form the final space. The results were found not to change significantly by including more Löwdin vectors.

At high temperatures results are calculated with great accuracy. This is easily understood because the quantity calculated at $\beta=0, \sum_{r=1}^{R} \sum_{i=0}^{M}\left\langle r|\hat{H}| \psi_{i}^{r}\right\rangle\left\langle\psi_{i}^{r} \mid r\right\rangle$, is rigorously equal to $\sum_{r=1}^{R}\langle r|\hat{H}| r\rangle$, and therefore the quality is mainly dependent on the sampling of the initial vectors (and not on the error of individual eigenvalue estimates). At low temperatures the results are dominated 


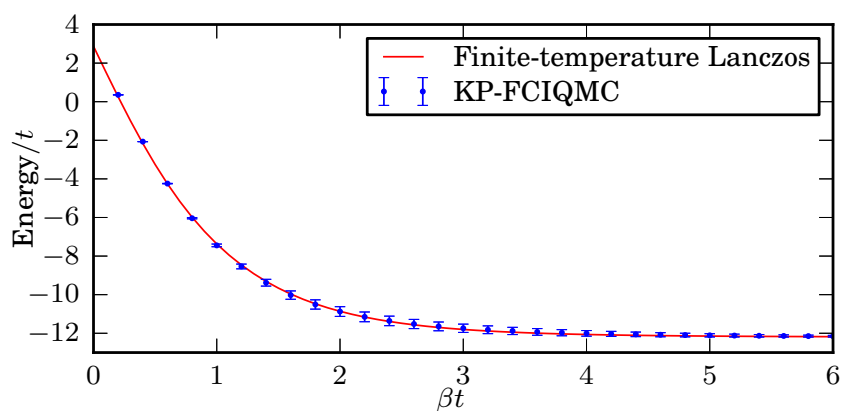

FIG. 1. $E(\beta)$ for the 12-site $1 \mathrm{D}$ Hubbard model at $U / t=1$ sampled with $\sim 2 \times 10^{3}$ walkers, with comparison FTLM. Error bars show standard deviation (note not standard error) over 10 independent calculations to demonstrate the spread of results. High and low temperature results are almost exact, whilst at intermediate temperatures, the variance in the stochastic sampling as well as systematic errors (such as from the non-linear diagonalization step, and finite $R$ ) increases the variation between runs. Simulation parameters were $\tau=0.01, n_{\mathrm{a}}=2.0$, and a deterministic space of double excitations $[35,36]$.

by the ground state, which has a large component in the sampled Krylov vectors and so is accurately calculated by KP-FCIQMC. However, at intermediate temperatures the errors are larger. The most significant source of error is in replacing an exact trace over $\{|n\rangle\}$ by an approximate one over $\{|r\rangle\}$ in Eq. 4.

In Figure 2, $E(\beta)$ for the two-dimensional 18-site Hubbard model at $U / t=1$ is presented. Including all symmetry sectors the Hilbert space dimension is $\approx 9 \times 10^{9}$, with the largest symmetry sector containing $\approx 1 \times 10^{8}$ determinants. Again, the space was undersampled, with $5 \times 10^{6}$ walkers used throughout, with $R=250$ and $M=20$, of which 12 Löwdin vectors are kept. Since FTLM was unfeasible, also plotted is a highly-accurate ground-state FCIQMC energy for comparison. A complete calculation took around $\sim 3000$ core hours. We find again that the high-temperature results have only a small variation between repeated calculations and we have a high degree of confidence here. At lower temperatures the confidence in the results is reduced, with possible systematic errors including initiator error, bias in the eigenvalue estimates and an insufficient choice of $R$.

Dynamical correlation functions:- To demonstrate the ability of KP-FCIQMC to calculate dynamical quantities, we first consider the following zero-temperature $k$ resolved single particle Green function, defined from Eq. 1 with $\hat{V}=\hat{A}=\hat{c}_{k \downarrow}^{\dagger}$. The corresponding spectral function, $A_{1}(k, \omega)=-\frac{1}{\pi} \Im[G(k, \omega)]$, defines the bandstructure of the material. The initial walker distribution is given by the perturbed ground state, $\hat{c}_{k}^{\dagger}\left|\Psi_{0}\right\rangle$, where $\left|\Psi_{0}\right\rangle$ is obtained from a prior ground-state FCIQMC calculation. This starting wavefunction ensures that on average the component of a particular eigenstate in any imaginarytime snapshot is proportional to its transition amplitude in the correlation function. This approach works particularly well for spectra dominated by a small number of states with large transition amplitudes. Because the transformation to the Löwdin basis introduces large er-

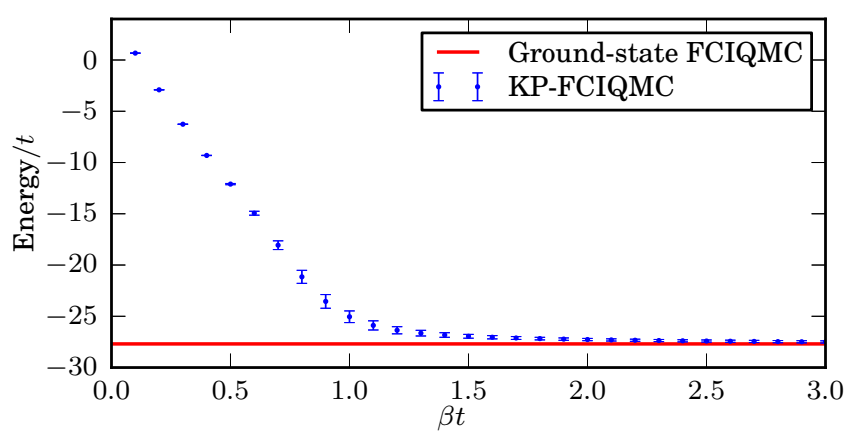

FIG. 2. $E(\beta)$ for the 18 -site $2 \mathrm{D}$ Hubbard model at $U / t=1$, with ground-state FCIQMC energy for comparison. 10 independent simulations were used to create the standard deviations shown as error bars. Simulation parameters were $\tau=0.01, n_{\mathrm{a}}=2.0$, and a deterministic space of double excitations.

rors if many states are kept (due to small overlap eigenvalues), we typically limit the number of Löwdin vectors to between 10 and 20, which limits the resolution of the spectrum. Furthermore, high-energy states die away rapidly in the Krylov vectors and so there tends to be significant stochastic errors associated with the calculation of such states. Although this limits the accuracy of KP-FCIQMC over a large energy range, we find that the method is capable of producing accurate spectra in the critical low-energy region and can accurately capture important features such as bandgaps.

Figure $3(\mathrm{a})$ presents $A_{1}(k, \omega)$ for the 14-site Hubbard model at $U / t=2$ with $\sim 10^{5}$ walkers, with $\boldsymbol{S}$ and $\boldsymbol{T}$ averaged over 10 repeats. 35 Krylov vectors were sampled and 10 Löwdin vectors were retained. A complete calculation for a given $k$-sector typically took only $\sim 6$ core hours. The results are compared to highly-accurate dynamical Lanczos results, using 100 Lanczos vectors. Figure 3(b) presents the local density of states, computed from the results in (a) via $A(\omega)=\frac{1}{N} \sum_{k} A(k, \omega)$. The KP-FCIQMC results give high accuracy for low-energy features, with sum rules and causality conditions exactly fulfilled. Errors on individual poles can be estimated by repeating results. By comparing eigenvalue estimates from 10 independent calculations, the bandgap was estimated as $0.96456(14) t$ compared to the exact value of $0.96378 t$.

We also consider the $s$-wave pair-pair dynamic correlation function, a two-body response property of significant relevance in the detection of superconducting quasiparticles. $\hat{V}$ is defined by the singlet pairing operator, $\Delta_{i}=\frac{1}{\sqrt{2}}\left(c_{i \uparrow} c_{i+1, \downarrow}-c_{i \downarrow} c_{i+1, \uparrow}\right)$, with $\hat{A}=\hat{V}$. In Fig. 4 we present results for this pairing spectrum $\left(A_{2}(\omega)\right)$ for the 10 -site Hubbard model at $U / t=1$, by computing all $k$-space contributions. The number of walkers was typically between $10^{3}$ and $10^{4}$. The initiator adaptation was not applied because the walker population is above the plateau[16] height for this system. No averaging of $\boldsymbol{T}$ or $\boldsymbol{S}$ over repeated calculations was performed. Once again, it is found that low-energy features are calculated accurately, but the quality decreases for higher energy regions of the spectrum.

Isolated excited states:- As a further application to 

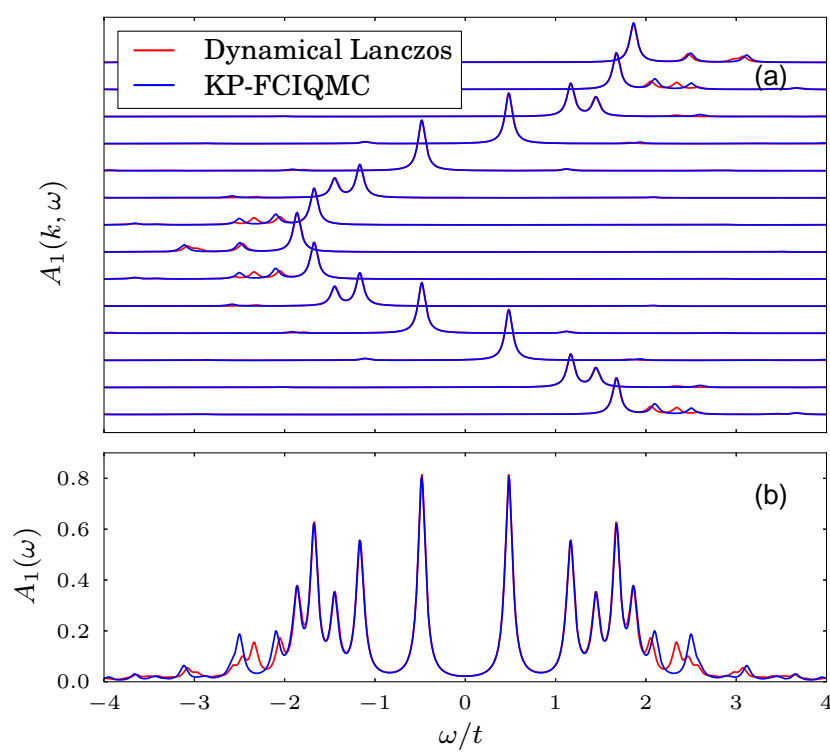

FIG. 3. (a) $A_{1}(k, \omega)$ from $k=-\frac{6}{7} \pi$ (bottom) to $k=\pi$ (top) for the 1D 14-site Hubbard model at $U / t=2$, compared to dynamical Lanczos. Poles coming from the ground state or low-lying excited states with large transition amplitudes are captured accurately. (b) The local density of states. The low-energy results are reproduced accurately by KP-FCIQMC while the qualitative behaviour is captured at high energies. Simulation parameters were $\tau=0.01, n_{\mathrm{a}}=3.0$, and a deterministic space of 50,000 determinants[35].

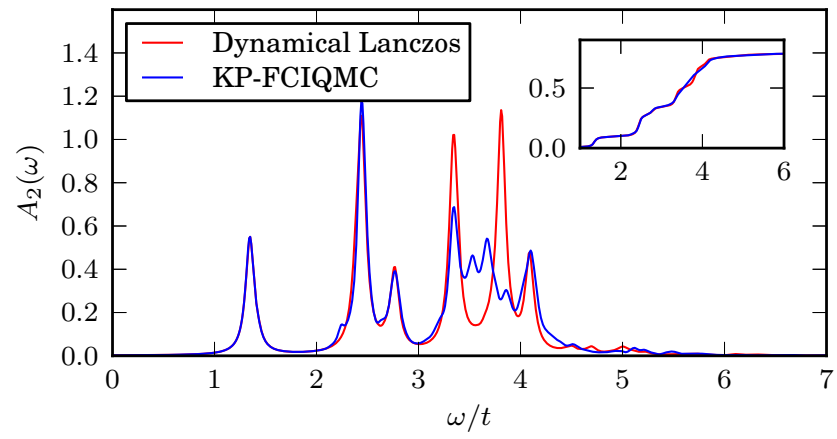

FIG. 4. $A_{2}(\omega)$ calculated for the 10-site Hubbard model with $U / t=1$, and compared to near-exact dynamical Lanczos. Inset shows integrated weight, $\int_{0}^{\omega} A_{2}\left(\omega^{\prime}\right) d \omega^{\prime}$. Simulation parameters were $\tau=0.01$, with a deterministic space of double excitations.

larger-scale $a b$ initio systems, we consider the all-electron ground and first excited state of Neon, in aug-cc-pVDZ and aug-cc-pVTZ basis sets[37]. We work in spaces where $M_{s}$ is constrained to be zero, but the total spin, $S^{2}$, is not. The $S=0$ and $S=1$ states are therefore both contained within the same symmetry sector, and the spin-gap can be directly targeted with KP-FCIQMC. The determinant space sizes with these two basis sets are $\sim 1.4 \times 10^{8}$ and $\sim 2.3 \times 10^{11}$, respectively. In order to ensure large components of the desired states in the sampled Krylov vectors, the initial wavefunction was created from a linear combination of trial estimates of the ground and first excited states at the inexpensive CISD level of theory.

KP-FCIQMC results are presented in table I, with

\begin{tabular}{cccccc}
\hline \hline & \multicolumn{3}{c}{$S=0$} & & \multicolumn{2}{c}{$S=1$} \\
\cline { 2 - 3 } \cline { 5 - 6 } Basis set & KP-FCIQMC & DMRG & & KP-FCIQMC & DMRG \\
\hline aug-cc-pVDZ & $-128.71143(4)$ & -128.71147 & & $-127.97787(5)$ & -127.97794 \\
aug-cc-pVTZ & $-128.8258(1)$ & -128.82514 & & $-128.109(1)$ & -128.10919 \\
\hline \hline
\end{tabular}

TABLE I. Results for the ground $(S=0)$ and first excited $(S=1)$ states of the Ne atom $\left(E_{h}\right)$, comparing KP-FCIQMC with DMRG (using $\mathrm{M}=500$ spin-adapted renormalised states for the larger basis) $[41,42] . \tau=0.001, n_{a}=3$ and a deterministic space of single and double excitations.

density matrix renormalisation group (DMRG) results for comparison. DMRG is a highly accurate algorithm, which can also be extended to thermal and spectral quantities, and so is a suitable choice for comparison[38-40]. KP-FCIQMC results and errors were estimated by averaging over 10 independent calculations. For the aug-ccpVDZ results, $2 \times 10^{5}$ walkers were used, while $2 \times 10^{6}$ walkers were used for the aug-cc-pVTZ basis, with each calculation taking around 100 core hours for this larger basis. Calculations used 35 Krylov vectors, with 10 Löwdin vectors retained, providing excellent agreement with DMRG.

Conclusion:- We have presented a novel approach to the calculation of excited state, spectral and thermal properties within the FCIQMC framework. In this approach the full Hamiltonian eigenvalue problem is projected into a stochastically sampled Krylov subspace, thus allowing finite-temperature and dynamical quantities to be calculated. Since the method exploits sparsity in the sampled wavefunctions, the stochastic dynamic avoids storing Krylov vectors in their entirety, rendering the approach scalable to systems sizes outside the range of the Lanczos method, although in practice this is likely to be restricted if attempting to probe high frequency spectral features.

\section{ACKNOWLEDGEMENTS}

G.H.B gratefully acknowledges funding from the Royal Society. N.S.B acknowledges Trinity College, Cambridge for funding. This work has been supported by the EPSRC under grant no. EP/J003867/1.

[1] W. M. C. Foulkes, L. Mitas, R. J. Needs, and G. Rajagopal, Rev. Mod. Phys. 73, 33 (2001)

[2] G. H. Booth, A. Grueneis, G. Kresse, and A. Alavi, Nature 493, 365 (2013)

[3] S. Sharma, T. Yanai, G. H. Booth, C. J. Umrigar, and G. K.-L. Chan, J. Chem. Phys. 140, 104112 (2014)

[4] Y. Virgus, W. Purwanto, H. Krakauer, and S. Zhang, Phys. Rev. B 86, 241406 (2012)

[5] S. Sorella, Y. Otsuka, and S. Yunoki, Sci. Reps. 2, 992 (2012)

[6] S. R. White, D. J. Scalapino, R. L. Sugar, and N. E. Bickers, Phys. Rev. Lett. 63, 1523 (1989) 
[7] M. Motta, D. E. Galli, S. Moroni, and E. Vitali, J. Chem. Phys. 140, 024107 (2014)

[8] A. W. Sandvik and J. Kurkijarvi, Phys. Rev. B 43, 5950 (1991)

[9] M. Jarrell and J. E. Gubernatis, Phys. Rep. 269, 133 (1996)

[10] E. Gull, P. Werner, A. Millis, and M. Troyer, Phys. Rev. B 76, 235123 (2007)

[11] A. J. Williamson, R. Q. Hood, R. J. Needs, and G. Rajagopal, Phys. Rev. B 57, 12140 (1998)

[12] D. M. Ceperley and B. Bernu, J. Chem. Phys. 89, 6316 (1988)

[13] S. Ten-no, J. Chem. Phys. 138, 164126 (2013)

[14] G. H. Booth and G. K.-L. Chan, J. Chem. Phys. 137, 191102 (2012)

[15] G. H. Booth, A. J. W. Thom, and A. Alavi, J. Chem. Phys. 131, 054106 (2009)

[16] J. S. Spencer, N. S. Blunt, and W. M. C. Foulkes, J. Chem. Phys. 136, 054110 (2012)

[17] J. Jaklic and P. Prelovsek, Phys. Rev. B 49, 5065 (1994)

[18] P. Prelovšek and J. Bonča, Strongly Correlated Systems (Springer Berlin Heidelberg, 2013) pp. 1-30

[19] P. E. Dargel, A. Woellert, A. Honecker, I. P. McCulloch, U. Schollwoeck, and T. Pruschke, Phys. Rev. B 85, 205119 (2012)

[20] A. Hams and H. De Raedt, Phys. Rev. E 62, 4365 (2000)

[21] S. Sugiura and A. Shimizu, Phys. Rev. Lett. 111, 010401 (2013)

[22] J. J. Shepherd, G. E. Scuseria, and J. S. Spencer, Phys. Rev. B 90, 155130 (2014)

[23] In FCIQMC the propagator $\hat{P}=1-\tau(\hat{H}-S 1)$ is used, where $\tau$ is a small timestep and $S$ is allowed to vary based on the walker population, $\sum_{i}\left|q_{i}\right|$, where $q_{i}$ is the walker weight on site $i$. See ref.[15] for more details.

[24] M. Caffarel, F. X. Gadea, and D. M. Ceperley, Europhys. Lett. 16, 249 (1991)

[25] S. Zhang and M. H. Kalos, J. Stat. Phys. 70, 515 (Feb 1993)

[26] N. S. Blunt, T. W. Rogers, J. S. Spencer, and W. M. C. Foulkes, Phys. Rev. B 89, 245124 (2014)
[27] C. Overy, G. H. Booth, N. S. Blunt, J. J. Shepherd, D. Cleland, and A. Alavi, J. Chem. Phys. 141, 244117 (Nov 2014)

[28] In practice, we sample the wavefunction with a linearly increasing number of iterations between selections, such that vectors during early iterations are sampled with finer resolution, where the wavefunction is rapidly changing and contains significant projections onto excited eigenstates.

[29] See Supplemental Material [URL], which includes Refs. [30-31]

[30] Z. Bai, J. Demmel, J. Dongarra, A. Ruhe, and H. van der Vorst, Templates for the Solution of Eigenvalue Problems: A Practical Guide (SIAM, Philadelphia, 2000)

[31] J. Cullum and R. Willoughby, Lanczos algorithms for large symmetric eigenvalue computations, Vol. 2 (Birkhäuser, Boston, 1985)

[32] T. Iitaka and T. Ebisuzaki, Phys. Rev. E 69, 057701 (2004)

[33] J. Schnack and O. Wendland, Eur. Phys. J. B. 78, 535 (Dec 2010)

[34] O. Hanebaum and J. Schnack, Eur. Phys. J. B. 3, 194 (Sep 2014)

[35] F. R. Petruzielo, A. A. Holmes, H. J. Changlani, M. P. Nightingale, and C. J. Umrigar, Phys. Rev. Lett. 109, 230201 (2012)

[36] D. M. Cleland, G. H. Booth, and A. Alavi, J. Chem. Phys. 134, 024112 (2011)

[37] T. Dunning, J. Chem. Phys. 90, 1007 (1989)

[38] S. R. White, Phys. Rev. Lett. 69, 2863 (1992)

[39] S. R. White and A. E. Feiguin, Phys. Rev. Lett. 93, 076401 (2004)

[40] A. E. Feiguin and S. R. White, Phys. Rev. B 72, 220401(R) (2005)

[41] R. Olivares-Amaya, W. Hu, N. Nakatani, S. Sharma, J. Yang, and G. K.-L. Chan, J. Chem. Phys. 142, 034102 (2015)

[42] S. Sharma and G. K.-L. Chan, J. Chem. Phys. 136, $124121(2012)$ 